\title{
Methanol Interactions with Nopinone Surfaces during Phase Evolution
}

Xiangrui Kong ${ }^{*}$, Josip Lovrić ${ }^{2}$, Sofia M. Johansson ${ }^{1}$, Nønne L. Prisle ${ }^{2}$, and Jan B. C. Pettersson $^{1 *}$

${ }^{1}$ Department of Chemistry and Molecular Biology, Atmospheric Science, University of Gothenburg, SE-41296, Gothenburg, Sweden

${ }^{2}$ Nano and Molecular Systems research unit, University of Oulu, 90014, Oulu, Finland 


\begin{abstract}
Organic-organic interactions play important roles in the secondary organic aerosol formation, but the interactions are complex and poorly understood. Here we use environmental molecular beam experiments combined with molecular dynamics simulations to investigate the interactions between methanol and nopinone, as atmospheric organic proxies. In the experiments, methanol monomers and clusters are sent to collide with three types of surfaces, i.e., graphite, thin nopinone coating on graphite and multilayer surfaces, at temperatures between $140 \mathrm{~K}$ and $230 \mathrm{~K}$. Methanol monomers are efficiently scattered from the graphite surface, whereas the scattering is substantially suppressed from nopinone surfaces. The desorption from the three surfaces is similar, suggesting that all the surfaces have weak or similar influences on the methanol desorption. The molecular dynamics results show that upon collisions the methanol clusters shatter, and the shattered fragments quickly diffuse and recombine to clusters. The desorption involves a series of processes, including detaching from clusters and desorbing as monomers. The experimental results also show that all trapped methanol molecules completely desorb within a short experimental time scale at temperatures of $180 \mathrm{~K}$ and above. At lower temperatures, the desorption rate decreases, and a long experimental time scale is used to resolve the desorption, where three desorption components are identified. The fast component is beyond the experimental detection limit. The intermediate component exhibits multi-step desorption character and has an activation energy of $E_{\mathrm{a}}=0.18$ $\pm 0.03 \mathrm{eV}$, in good agreement with simulation results. The slow desorption component is related to diffusion processes due to the weak temperature dependence.
\end{abstract}

\title{
Keywords
}

Environmental molecular beam, molecular dynamics, phase transition, secondary organic aerosol, sorption kinetics 


\section{Introduction}

Organic compounds are omnipresent in the atmosphere and continuously undergoing complex interactions and reactions. ${ }^{1}$ A sound understanding of atmospheric chemistry and phase transitions requires not only the individual oxidation pathways from secondary organic aerosol (SOA) precursors but also needs to take account of interactions between the multitude of different organics formed. ${ }^{2}$ However, the knowledge of the organic-organic interactions, especially on the mechanism at a molecular level, is limited and therefore largely missing from current climate models and leads to significant uncertainties when evaluating climate changes. ${ }^{3}$

Biogenic volatile organic compounds (BVOCs) have been recognized as important precursors for SOA as their volatilities can be significantly reduced after oxidization. ${ }^{4}$ For example, $\beta$ pinene is a common BVOC emitted by coniferous vegetation and is considered as a major source of aerosol particles. ${ }^{5}$ As one of the major oxidation products from $\beta$-pinene, ${ }^{6}$ nopinone has been found in both gas and particle phase of SOA, ${ }^{7}$ which motivated the recent studies on water-nopinone interactions, where nopinone was considered as a biogenic SOA proxy. Due to the cyclic structure, nopinone has a relatively high solubility in water compared to other ketones with similar sizes but linear structures. ${ }^{8}$ On water surface, the uptake of nopinone was found to be reversible with a wetted-wall flow tube reactor. ${ }^{9}$ On nopinone surface, the uptake of water molecules and the detailed molecular dynamics and kinetics were studied by an environmental molecular beam (EMB) technique. ${ }^{10,11}$ The thin coating and multilayer of nopinone show different water uptake ability, where more water was taken up by the multilayer. Molecular dynamics (MD) simulations show that bulk diffusion is very limited, and that the enhanced water uptake is instead mainly caused by water molecules that more easily find strong binding states on the multilayer surface than on the thin coating. ${ }^{11}$

Apart from water vapor-surface interactions, the organics-surface interactions are also important because of the abundance of organic vapors in the atmosphere. A recent EMB-MD study shows that methanol monomers and clusters are efficiently trapped on a graphite surface, and the trapped molecules rapidly diffuse along the surface to find other clusters or form new clusters at low surface coverages. ${ }^{12}$ Methanol molecules form hydrogen bonds within the clusters internally, which strengthens the structure and the stability of clusters. Herrera et al. ${ }^{13}$ reported that small methanol clusters are preferably formed on graphite because of the interaction of hydrogen bonds. Apart from the graphite surface, various organic surfaces have 
also been studied by EMB,${ }^{14}$ including alcohols, ${ }^{15-19} \operatorname{ketones}^{10,11}$ and carboxylic acids,${ }^{20}$ but the interactions of organic surfaces with another organic gas species have never been studied by the EMB.

The combination of EMB and MD has been shown to be an effective approach to investigate the gas uptake and sorption kinetics of gas interactions with various surfaces. ${ }^{11,12,16,17}$ In this study, we look into the kinetics of methanol monomers and clusters on nopinone surfaces (thin coating and multilayer) by the EMB experiments and the MD simulations. The inter-species interactions are compared to the internal interactions within phases, which reveals the important parameters determining the thermodynamics and kinetics of mixed organic systems.

\section{Methodology}

\subsection{EMB Experiments and Data Analysis}

The EMB method is used to investigate the dynamics and kinetics of methanol interactions with nopinone surfaces. The experimental setup has been previously described in detail, ${ }^{21,22}$ and consists of a three-chamber differentially pumped beamline. Beam pulses are generated from a beam source, and a portion of the pulsed gas flow travels through a skimmer (diameter $=1 \mathrm{~mm}$ ) to form a directed low-density beam of molecules in the forward direction. The nozzle of the beam source is kept at the room temperature, and no methanol condenses on the nozzle. The beam is composed of methanol and helium, where a helium flow passes through a methanol reservoir to pick to the methanol molecules and clusters. The beam is modulated by a chopper with a frequency of $120 \mathrm{~Hz}$ (duty time 50\%) for the short experimental time scale (10 ms) and with a frequency of $8 \mathrm{~Hz}$ (duty time $50 \%$ ) for the long experimental time scale $(60 \mathrm{~ms})$. When measuring the beam using a quadrupole mass spectrometer (QMS), the most intense peak in the mass spectrum is $m / z=31\left(\mathrm{CH}_{3} \mathrm{O}^{+}\right)$. Other major peaks are $m / z=33,65,97,129,161$, corresponding to $\mathrm{H}^{+}\left(\mathrm{CH}_{3} \mathrm{OH}\right)_{n}$ with $\mathrm{n}=1-5$, i.e., the methanol clusters in the beam. It is not possible to determine the exact size of clusters due to unknown fragmentation during ionization, but the clusters are seemingly relatively small, ranging from a few molecules to tens of molecules per cluster, based on the facts of the relatively low source pressure, the shape of the nozzle that is not optimized for cluster production, and the relatively similar intensities of monomers and clusters. 
The measured Time-of-Flight (ToF) distributions of $\mathrm{m} / \mathrm{z}=31\left(\mathrm{CH}_{3} \mathrm{O}^{+}\right)$and $\mathrm{m} / \mathrm{z}=33$ $\left(\mathrm{H}^{+} \mathrm{CH}_{3} \mathrm{OH}\right)$ intensities show that the clusters travel with lower velocities than monomers, as known from earlier studies. The beam is supersonic, and the estimated mean velocity for monomers is about $1510 \mathrm{~m} / \mathrm{s}$, corresponding to the kinetic energy (KE) of $0.38 \mathrm{eV}$. The beam pulses then collide with a surface centered in an environmental chamber, and the outgoing flux is measured by a rotatable differentially pumped QMS for ToF measurements. Ions generated by electron bombardment in the QMS are detected by a multi-channel-scaler with a dwell time of $10 \mu \mathrm{s}$. The studies presented here are performed with a highly-oriented polycrystalline graphite (HOPG) substrate surface $(12 \times 12 \mathrm{~mm}$ surface, Advanced Ceramics Corp., grade $\mathrm{ZYB})$, which is cleaned by keeping it at $600 \mathrm{~K}$ before and after experiments. The nopinone surfaces are prepared by dosing nopinone ((1R)-(+)-Nopinone, 98\% Sigma-Aldrich Co.) vapor through a leak valve, where the nopinone multilayer is maintained at a thickness of $\sim 1 \mu \mathrm{m}$. The properties and thickness of the condensed nopinone layer are monitored by the helium in the beam and a laser $(670 \mathrm{~nm})$ interferometry. The thickness of the multilayer is monitored by the laser, whereas the thin nopinone coating cannot be seen by the laser but are detected by helium scattering. ${ }^{11}$

The ToF distributions are fitted to resolve the kinetics and dynamics of the interactions between the impacting methanol molecules/clusters with the nopinone surfaces. Typically, impinging molecules can be scattered inelastically or thermally trapped, thus these components are sought for in our analysis. Non-linear least squares fits are carried out to deconvolute the inelastic scattering (IS) and thermal desorption (TD) components. The IS component is represented by a velocity dependent function, ${ }^{23}$

$$
I_{I S}(v(t))=C_{i} v(t)^{4} \exp \left[-\left(\frac{v(t)-\bar{v}}{\sqrt{\frac{2 k_{B} T_{I S}}{m}}}\right)^{2}\right],
$$

where $C_{i}$ is a scaling parameter, $v$ is the velocity calculated from the molecular arrival time, $\bar{v}$ is the average velocity, $k_{B}$ is the Boltzmann constant, $m$ is the molecular mass of methanol, and $T_{I S}$ is a free parameter representing the IS velocity spread.

The TD distributions are each combination of two components: (i) a velocity distribution that relates desorption to molecular excitation based on the surface temperature, 


$$
I_{T D 1}(v(t))=C_{j} v(t)^{4} \exp \left[-\left(\frac{v(t)}{\sqrt{\frac{2 k_{B} T_{S}}{m}}}\right)^{2}\right],
$$

and (ii) another distribution related to the desorption rates,

$$
I_{T D 2}=C_{j} e^{-k t}
$$

where $C_{j}$ is a free scaling factor, $T_{s}$ is the surface temperature, $k$ is the fitted desorption rate coefficient, and $t$ is time. $I_{T D 1}$ shows the velocity spread of the TD flux, and $I_{T D 2}$ accounts for the exponential decay of ToF distributions. Thus, the TD distributions are calculated as a convolution of these two components.

\subsection{Simulations}

MD simulations are performed to characterize methanol monomer and cluster collision with solid nopinone surface. The classical mechanics Gromos force field ${ }^{24}$ optimized for small molecules in condensed phases is employed to model a nopinone crystal. The force field is implemented in the GROMACS package ${ }^{25}$ with the help of the ATB database. ${ }^{26}$ The original ATB GROMOS charges are unable to reproduce melting processes, therefore a new set of charges is calculated based on $a b$ initio calculations. $\operatorname{RESP}^{27}$ point charges fixed on atomic position are fitted to best reproduce the electrostatic field induced by the electronic density of isolated nopinone molecule calculated on BLYP-D3 level of theory while having electrons spanned over the DZVP basis set.

The equations of motion are integrated using the leap-frog approach ${ }^{28}$ and LINCS constrain algorithm ${ }^{29}$ which makes it possible to use a 2 fs time step. A cut off distance of $1.8 \mathrm{~nm}$ is applied for the short-range interactions, and long-range electrostatic interactions are treated using the particle mesh Ewald method. ${ }^{30}$ Nopinone crystal temperature is held at the desired value using the V-rescale algorithm ${ }^{31}$ with a coupling time of $0.1 \mathrm{ps}$.

The nopinone crystal is modelled based on the powder X-ray diffraction data by Palin et al. ${ }^{32}$ The crystal structure is freely available at Cambridge structural database. ${ }^{33}$ An infinite crystal is created by duplicating a unit cell in $x, y$ and $z$ directions. After a minimization with the steepest descent algorithm, the crystal is equilibrated in the NPT ensemble at $220 \mathrm{~K}$ for $10 \mathrm{~ns}$. The simulation system consists of $\sim 10000$ atoms placed in orthorhombic box stretched by $a$, $b, c$ vectors sized $3.91 \times 4.18 \times 7.53 \mathrm{~nm}$. The relaxed crystal is then used to form a slab considering the most energetically favorable exposed crystal surface, thus the crystal is cut 
between two nopinone bilayers. The simulation box is then extended up to $2 \mathrm{~nm}$ along the $z$ direction on each side to avoid image interactions between the slabs. After producing the nopinone solid slab, methanol interactions with the nopinone surface are studied at $220 \mathrm{~K}$. Simulations are designed to resemble the experimental conditions. Monomers and methanol clusters are present in the beam, therefore both two cases are modelled. Methanol is also modelled with the Gromos force field ${ }^{24}$ and refined RESP $^{27}$ point charges are applied as previously explained for nopinone.

First, to model monomer collision, single methanol molecules are sent towards the surface with an incident kinetic energy equal to $0.24 \mathrm{eV}\left(1700 \mathrm{~m} \mathrm{~s}^{-1}\right)$ and with an incident angle of $45^{\circ}$ with respect to the surface normal direction. Methanol is decoupled from the thermostat to avoid influencing collision dynamics. The initial $(x, y)$ positions of methanol molecules are randomly chosen at a distance of $1 \mathrm{~nm}$ from the surface and 2000 trajectories are propagated at each studied surface temperature. Methanol clusters on nopinone surface system are also investigated. Initially, 10 methanol molecules are packed in a cluster and equilibrated at $180 \mathrm{~K}$ in the NVT ensemble. Thereafter the cluster is placed $1 \mathrm{~nm}$ above the solid nopinone surface at $220 \mathrm{~K}$ and consecutively send towards the surface at a velocity of $1700 \mathrm{~m} \mathrm{~s}^{-1}$ and an incident angle of $45^{\circ}$ with respect to the surface normal direction while being decoupled from the thermostat.

\section{Results and Discussions}

\subsection{EMB Experiments}

\subsubsection{Monomer and Cluster Beam Profiles}

The molecular beam used in EMB experiments contains methanol monomers and methanol clusters of various sizes. ${ }^{12}$ The beam composition is varied by shifting the beam source offtime $\left(\mathrm{t}_{\text {off }}=2.2\right.$ or $\left.2.4 \mathrm{~ms}\right)$. Figure 1a shows the profiles when $\mathrm{t}_{\mathrm{off}}=2.4 \mathrm{~ms}$, where both clusters and monomers appear as peaks. This feature allows for the study of the interactions between monomers and the surfaces. All the peaks have independent shapes, indicating that the clusters only contributed to their own primary $\mathrm{m} / \mathrm{z}$ but not to others, i.e., otherwise the lower $\mathrm{m} / \mathrm{z}$ would show contributions from the higher $\mathrm{m} / \mathrm{z}$. The molecular velocities depend on the monomer or cluster masses, so the lightest mass, i.e., monomers $(m / z=31)$, appears at the beginning of the Time-of-Flight spectrum, and the heavier clusters arrive later. Another beam setting is the cluster beam ( $\mathrm{t}_{\mathrm{off}}=2.2 \mathrm{~ms}$ ), and the beam profile is shown in Figure 1b. In this case, the beam 
is dominated by clusters with a square-like beam shape, which enables accurate fittings to the ToF results.

Figure 1c shows an example of the methanol flux from a $200 \mathrm{~K}$ graphite surface after the beamsurface collisions, where the monomer and cluster beams are compared. For the cluster beam $\left(t_{\text {off }}=2.2 \mathrm{~ms}\right)$, the flux appears as a smooth peak, while for the monomer peak $\left(\mathrm{t}_{\text {off }}=2.4 \mathrm{~ms}\right)$, because it contains a fraction of monomers that travels faster, this results in an inelastic scattering (IS) component appearing at the beginning of the ToF spectrum. This indicates that
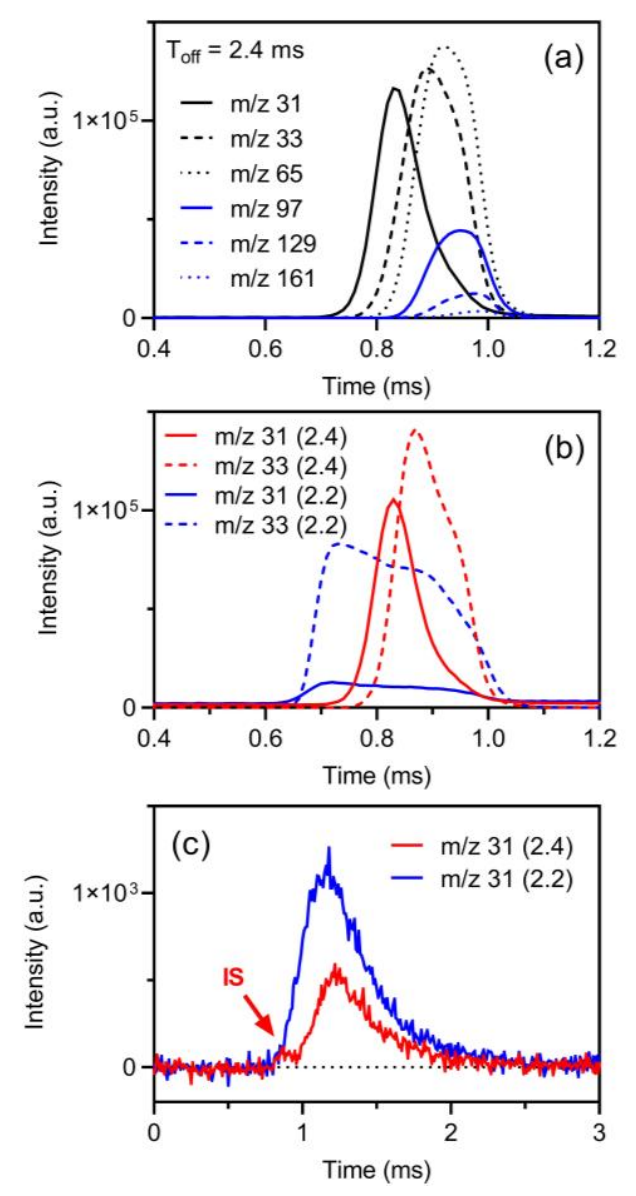

Figure 1 (a) beam profiles of monomers and clusters of different sizes; ( $b$ ) beam profiles of $\mathrm{m} / \mathrm{z}=31$ and $\mathrm{m} / \mathrm{z}=33$ for monomer beam $\left(T_{\text {off }}=2.4 \mathrm{~ms}\right)$ and cluster beam $\left(T_{\text {off }}=2.2 \mathrm{~ms}\right) ;(\mathrm{c})$ ToF of monomer flux from graphite at $200 \mathrm{~K}$, using monomer beam $\left(T_{\text {off }}=2.4 \mathrm{~ms}\right)$ and cluster beam $\left(T_{\text {off }}=2.2 \mathrm{~ms}\right)$.

methanol monomers are scattered from the surface while the clusters are not. Under the experimental incident kinetic energy, no clusters flux $(\mathrm{m} / \mathrm{z}, 33$ and 65) are detected from any surfaces, indicating that methanol is scattered or desorbs only as monomer.

\subsubsection{Inelastic Scattering and Thermal Desorption}

When methanol monomers impinge on the surfaces of nopinone coating or multilayer, the scattered component is substantially different compared to that from a graphite surface. Figure 
2a shows that the IS (shown in blue dashed lines) from graphite vanishes from its original position in both the nopinone cases. This indicates that methanol molecules transfer energy efficiently with the nopinone surfaces, which is comparable to the case of water molecules
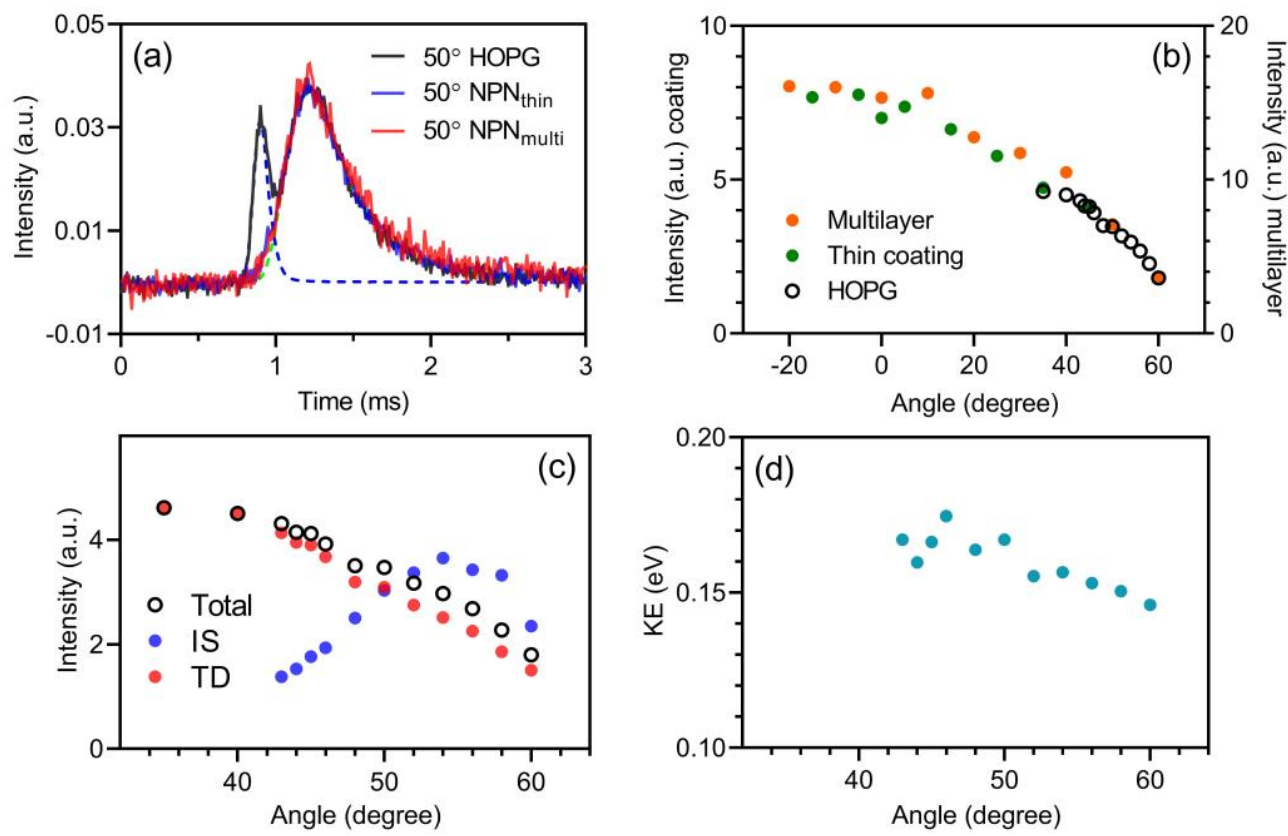

Figure 2 (a) ToF spectra of methanol flux from graphite, nopinone coating and multilayer surfaces after the monomer beam collisions. The surface temperatures are $210 \mathrm{~K}$, and the detection angle is $50^{\circ}$ in respect to the surface normal. (b) Angular distributions of total methanol flux from graphite, nopinone coating and multilayer at $210 \mathrm{~K}$. (c) Angular distributions of IS and TD components, and the IS intensity is multiplied by

a factor of 8 to be showed in the same scale. (d) Angular distributions of kinetic energy of IS molecules.

impinging on nopinone surfaces that has been studied previously. ${ }^{10,11}$ The methanol-nopinone surface interactions have also been investigated by MD simulations, where both inelastic scattering and fast thermal desorption are found (Figure S2). Other than that, the desorption components remained almost identical in the three cases. Because the IS components are subtle, the angular distribution of total flux from nopinone surfaces are basically cosine shaped (Figure $2 b)$, which is a characteristic for TD.

Figure $2 \mathrm{c}$ shows the angular distributions of IS and TD flux intensity from the graphite surface. The IS intensity peaked at around $54^{\circ}\left(c . f\right.$., incident angle $=45^{\circ}$ ), and the IS peaking at wide angles is common for molecules scattering on smooth surfaces. ${ }^{34}$ Figure $2 d$ displays the variation of $\mathrm{KE}(0.14-0.18 \mathrm{eV})$ of the IS molecules, accounting for $37-47 \%$ of the initial KE. The KE decreases as the scattering angle increases, which is typical for inelastically scattered molecules from graphite surfaces. ${ }^{34-36}$ The IS components cannot be fitted for the observation angles $<43^{\circ}$ because the signal is too small.

\subsubsection{Desorption Kinetics}


The desorption fluxes from the graphite, thin nopinone coating and multilayer surfaces show similar temperature dependence. Figure 3 a shows that the desorption fluxes increase with temperature at temperatures below $180 \mathrm{~K}$. Above $180 \mathrm{~K}$, the desorption intensities do not change with temperature, indicating that all trapped methanol molecules have desorbed within the detection time scale $(10 \mathrm{~ms})$. It is interesting to see that neither the nopinone coating nor multilayer influences the methanol uptake compared to the graphite surface. This differs from the recently studied water - nopinone system, where water uptake is enhanced on nopinone surfaces compared to on graphite. ${ }^{11}$ The insensitivity of surfaces suggests that the desorption processes are dominated by internal methanol-methanol interactions within clusters. Notably, the melting point of methanol is $\sim 175 \mathrm{~K}$ (marked with a dashed purple line in Figure $3 \mathrm{a}$ ), which separates the desorption flux trend into a temperature-sensitive region and a temperatureinsensitive region.
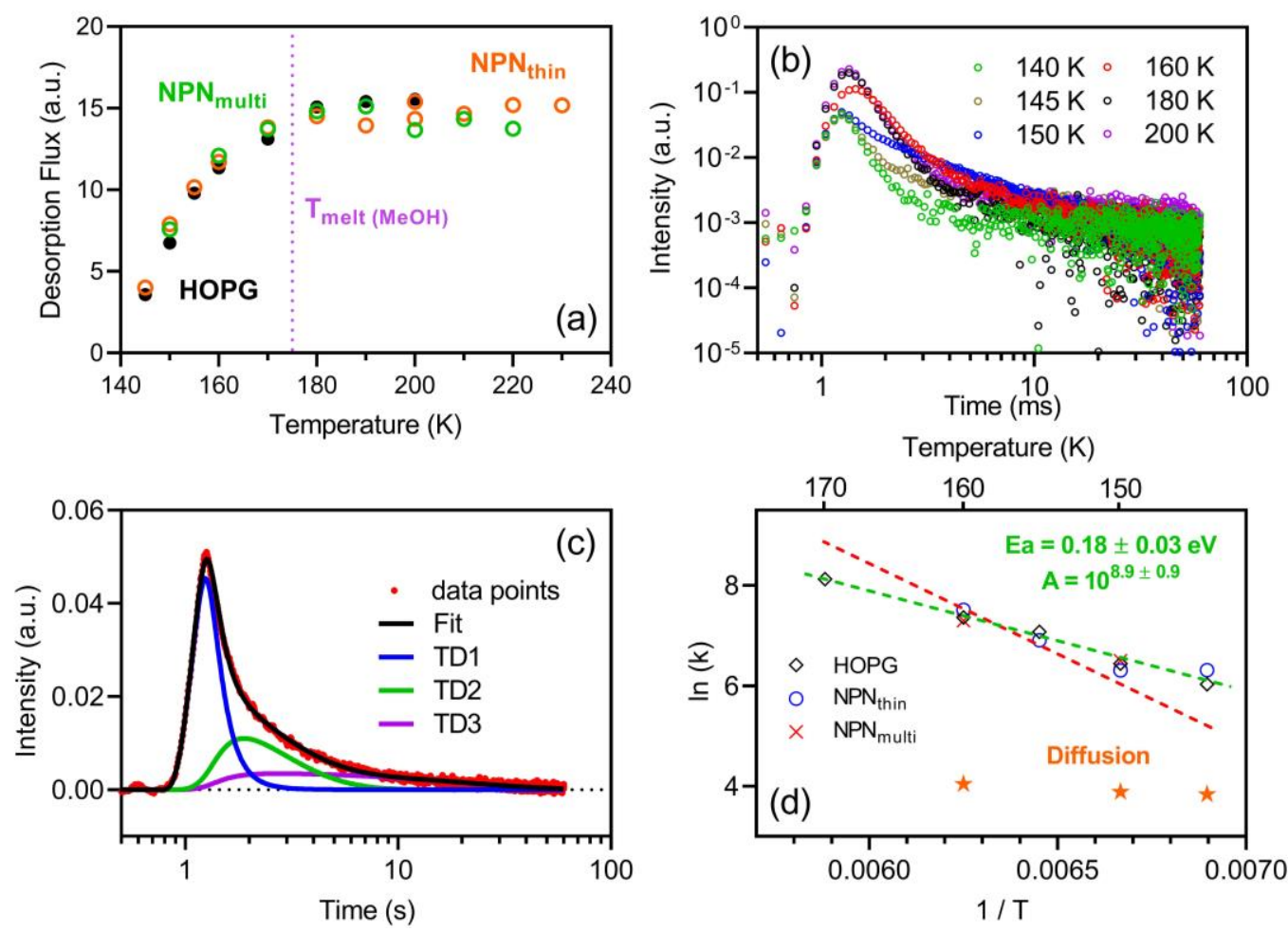

Figure 3 (a) Temperature-dependent desorption flux from graphite, thin nopinone coating and multilayer. The intensities have been normalized to beam intensities. The melting point of methanol bulk is marked as the purple dotted line. The experimental time scale is $10 \mathrm{~ms}$. (b) ToF of long experiments (60 ms) at various temperatures. (c) ToF measured at $150 \mathrm{~K}$, fitted by three TD components. (d) Arrhenius plot for the TD2 component at the temperature range between 140 - $170 \mathrm{~K}$. The green dashed line is the best fitting to the data points, where Ea $=0.18 \pm 0.03 \mathrm{eV}$ and $A=1 \cdot 10^{8.9 \pm 0.9}$. The red dashed line is the best fitting with the assumption of $A=1 \cdot 10^{13}$.

The detection time scales may potentially influence the quantification of desorbed fractions, especially at lower temperatures. ${ }^{37}$ To account for this, longer experiments with $60 \mathrm{~ms}$ scanning 
time are carried out and the ToF spectra are shown in Figure 3b. Apparently, at most temperatures the desorption does not end by $60 \mathrm{~ms}$, which confirms the existence of slow desorption channels. Three-TD fittings are used to fit the $60 \mathrm{~ms}$ ToF at the temperature range between 140 - $170 \mathrm{~K}$ (Figure 3c). Two-TD fittings are also attempted but there were always noticeable residues between fittings and actual data points, showing that it is necessary to have three-TD components (See Figure S1 for example). Note that the ToF at temperatures higher than the methanol melting points, i.e., $180 \mathrm{~K}$ and $200 \mathrm{~K}$, cannot be fitted using three TD components but by only one (the fastest desorption, TD1). The absence of the other two TD components is likely due to the solid-liquid phase transition of methanol on the surface, i.e., the TD2 and TD3 are processes occurring on non-liquid surfaces.

As the rates of TD1 are always beyond the EMB detection limit $\left(\mathrm{k}>10^{5}\right)$, only the resolvable rates of TD2 and TD3 are presented in the Arrhenius plot (Figure 3d). The slow TD (TD3) is likely associated to diffusion related processes due to the weak temperature dependence. As for the intermediate desorption (TD2), no differences are found among the three kinds of surfaces. The activation energies are around $0.18 \pm 0.03 \mathrm{eV}$ with the pre-exponential factor $(A)$ of $1 \cdot 10^{8.9 \pm 0.9}$. Such low activation energy is comparable to the binding energy of methanol monomer on graphite, ${ }^{38}$ but the low $A$ value indicates that the yielded activation energy is a result from complicated processes rather than a first-order desorption. For comparison, by artificially assigning the pre-exponential factor to the typical value for first order desorption ( $A$ $=1 \cdot 10^{13}$ ), the activation energy obtained from the constrained best fitting is then $0.31 \mathrm{eV}$ (red dashed line), but it is obviously deviating from the experimental data points.

\subsection{Simulations}

\subsubsection{Collision Dynamics}

The methanol-nopinone system is modelled by classical MD, where both monomers-surface and clusters-surface interactions are simulated. The nopinone crystal is constructed based on experimental data, ${ }^{32}$ which is characterized by bilayer structure having functional groups directed inside the bilayer (Figure 4a). The molecules within bilayers cohere through relatively week hydrogen bonds between carbonyl groups from one side and hydrogens atoms from the surrounding molecules. ${ }^{32}$ Weak van der Waals forces between bilayers are responsible for overall crystal structure cohesion. The melting point of nopinone has been experimentally determined to $260 \mathrm{~K}^{32}$ In this work, the investigated temperature is well below melting point, i.e., the simulations are conducted at $220 \mathrm{~K}$, so the nopinone has a crystalline structure. The 
nopinone surface is characterized by well-ordered hydrocarbon groups while having carbonyl groups generally inaccessible. The appearance of hydroxyl groups on the surface may be considered as surface defects, which appears as one of the surface molecules rotate for $180^{\circ}$ around one of the surface axes. Such hydrophilic sites on the surface influence the desorption dynamics of incoming molecules significantly. This is especially evident for small molecules, like water, ${ }^{11}$ which can rapidly diffuse on smooth nopinone surface and eventually bind strongly for this site.

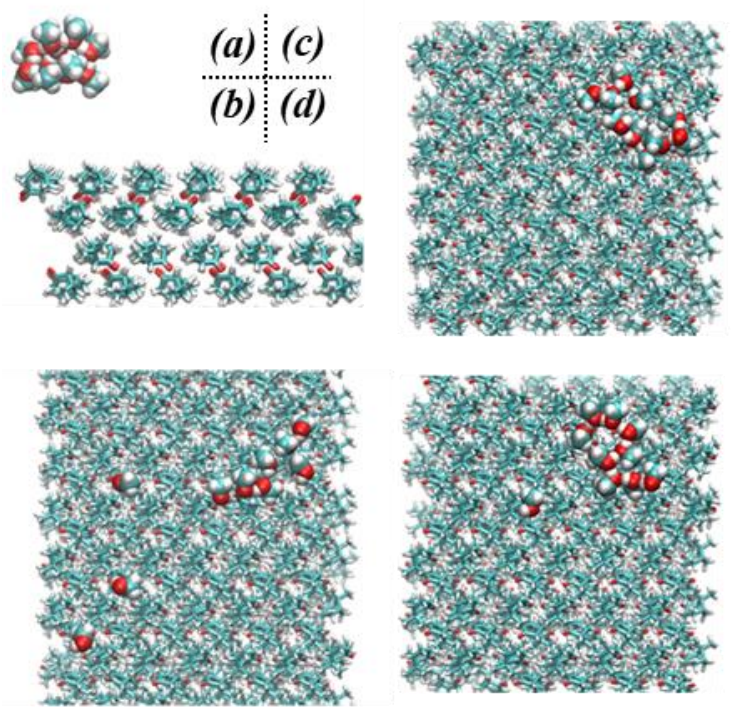

Figure 4 Snapshots of a ten-molecule methanol cluster colliding with solid nopinone surface at $200 \mathrm{~K}$. Snapshot taken at a) $t=0 \mathrm{ps} ; b) t=24 \mathrm{ps} ; \mathrm{c}) t=108 \mathrm{ps} ; \mathrm{d}) t=154 \mathrm{ps}$.

Figure 4 presents snapshots from simulations taken on critical moments during methanol cluster-surface collisions. Figure 4a shows initial condition of simulation, where a 10-molecule cluster is placed $1 \mathrm{~nm}$ above the surface. Just after colliding with the surface and during the first $20-30$ ps as the cluster shatters and fragments on the surface (Figure 4b). Evidently, there is a significant number of methanol monomers loosely bound to the nopinone surface (Figure $4 c)$, which can easily undergo thermal desorption. In the next step, methanol molecules that do not undergo desorption within a period of $\sim 100 \mathrm{ps}$ form clusters on the surface again. Methanol molecules diffuse on surface efficiently (diffusion coefficient $\approx 5.35 \times 10^{-5} \mathrm{~cm}^{2} / \mathrm{s}$ ), which allows them to quickly find other methanol molecules or clusters and bind strongly. This is comparable to methanol monomers diffusion on graphite surface, even at very low surface coverages. ${ }^{12}$ Molecules inside clusters are more strongly bound and therefore not able to desorb directly. The molecules on the cluster edges are relatively weakly bound, and they can either directly desorb from clusters or go through a two-step process where they first detach from clusters (Figure $4 d$ ) and then desorb as monomers. 


\subsubsection{Cluster Evolution}

The evolution of clusters over time is presented from the aspect of binding energies distribution shown in Figure 5. The energy is calculated as the instantaneous interaction energy of one methanol molecule with the whole surrounding system including the nopinone surface and other methanol molecules (left panel), but without the nopinone surface (right panel). The distribution in the top panel is corresponding to isolated methanol clusters, which serves as a reference for the other two cases. All distributions in Figure 5 are characterized with a broad energy range indicating the complex nature of the system, which results in multistep dynamics. This agrees with the experimentally determined kinetics parameter, which indicates complex desorption mechanisms. Figure $5 \mathrm{~b}$ shows the snapshot $\sim 50 \mathrm{ps}$ after the collisions and the weakly bounded states are visible, as the energy distribution is shifted to the lower energies. The cluster undergo fast re-arrangements as seen in Figure $5 \mathrm{c}$ as the distribution is shifted to higher binding energies again. At approximately 2 ns after surface impact, methanol clusters are stable on the surface. The distribution profile in Figure 5d show the multiple components corresponding to several binding states in the clusters.

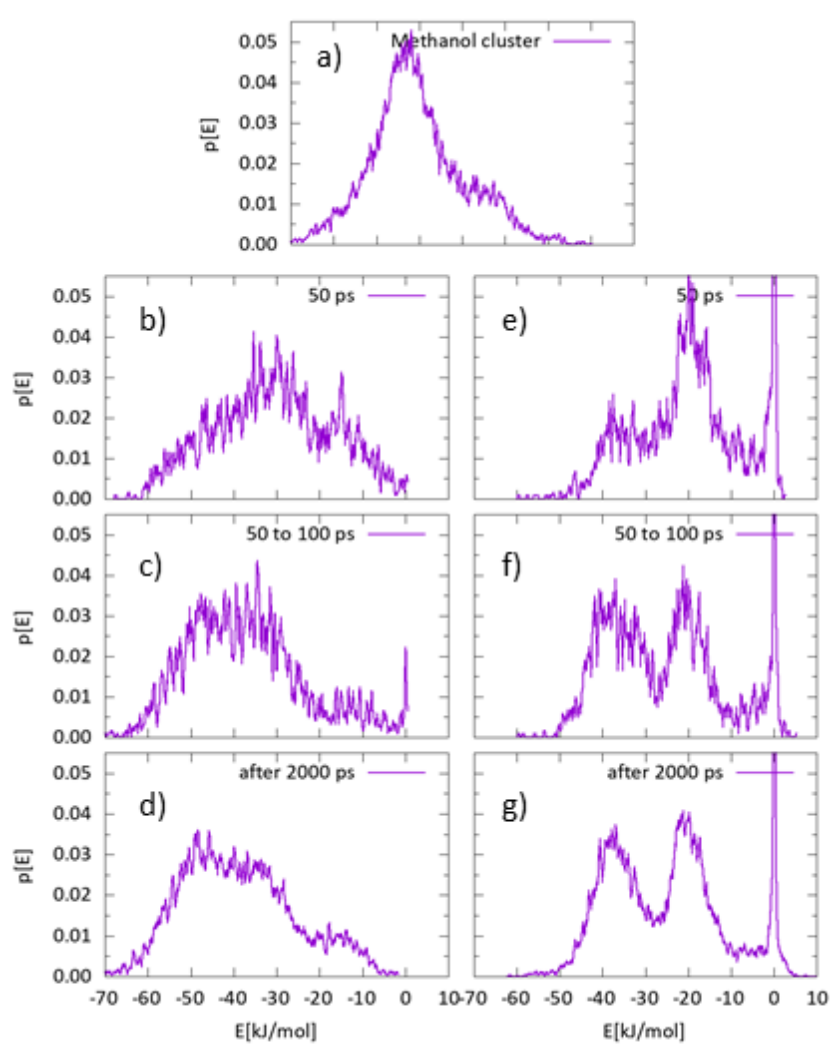

Figure 5 Probability distributions retrieved by histograming instantaneous binding energies between individual methanol molecules with surrounding molecules. (a) shows distribution inside isolated methanol clusters. On the left panel, from $(b)$ to $(d)$, are distributions correspond to binding energies between methanol molecules and the surrounding system including the nopinone surface. On the right side, from (e) to $(g)$, the nopinone surface is omitted from calculation of binding energy, thus showing exclusively interaction among methanol molecules. Time window for which distribution is calculated is visible as a legend in upper right corner of each individual plot. 
For comparison, the right-hand side panels show the energy distributions when the nopinone surface is omitted, thus only the methanol-methanol interactions are visible. Apparently, when there is no surface present, the energy distribution is more sharply peaked at $-40 \mathrm{~kJ} \mathrm{~mol}^{-1}(\sim 2$ hydrogen bonds) and $-20 \mathrm{~kJ} \mathrm{~mol}^{-1}$ ( 1 hydrogen bond) (Figure $5 \mathrm{~g}$ ), revealing two typical conformation configurations. One is stronger, inside of the cluster and the other, seen on the edges, is more probable to desorb. The difference between the left and right panels indicates the influence of the nopinone surface on the cluster binding states. The presence of a surface strengthens the binding energy of the methanol molecules due to the methanol-nopinone interactions, and the smoothing effect of the surface indicates the complexity of the system of methanol cluster on the nopinone surface. Nevertheless, inside cluster interaction energy is dominant in the overall energy distribution, especially when the cluster is equilibrated on the surface, and this supports the conclusion statement that desorption dynamics are mainly internally driven.

\subsubsection{Temperature Effects}

The methanol desorption from all the investigated surfaces (including a graphite surface, a thin nopinone coating on graphite and a solid nopinone surface) shows a uniform temperature dependence, indicating that all the surfaces either have similar or negligible influences on the desorption processes. To understand the temperature dependence of methanol desorption from nopinone surface, a methanol cluster consisting of 60 molecules is placed above the nopinone surface and equilibrated at different temperatures. Two distinct configurations of methanol clusters on top of the nopinone surface at two different temperatures (100 and $150 \mathrm{~K}$, respectively) are shown in Figure 6. At the lower temperature (Figure 6a), the methanol cluster retains a compact shape, and the methanol-methanol internal interactions (binding energy = $30.8 \mathrm{~kJ} \mathrm{~mol}^{-1}$ ) are stronger than the methanol-surface interactions (binding energy $=8 \mathrm{~kJ} \mathrm{~mol}^{-}$ ${ }^{1}$ ). At the higher temperature (Figure 6b), the methanol molecules spread on the nopinone
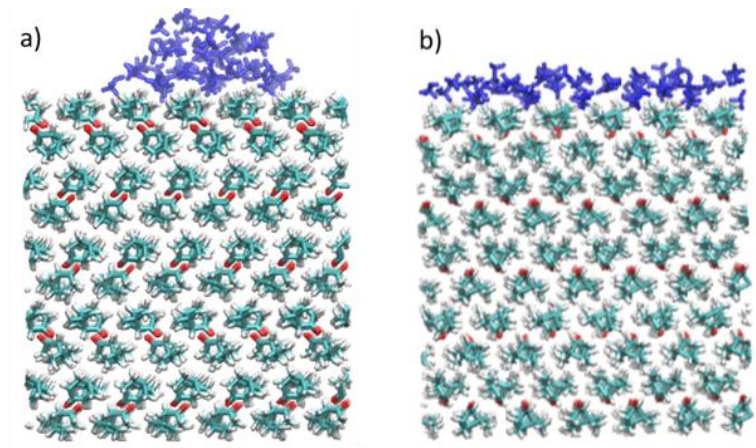

Figure 6. Configurations of methanol cluster (in blue) on the top of solid nopinone surfaces (green, red and white atom representation) at (a) $T=100 \mathrm{~K}$ and (b) $T=150 \mathrm{~K}$. 
surface, and the methanol-surface interactions become more significant (binding energy $=25$ $\mathrm{kJ} \mathrm{mol}^{-1}$ ) while the internal methanol-methanol interactions are weakened (binding energy $=$ $13 \mathrm{~kJ} \mathrm{~mol}^{-1}$ ). The total binding energies of methanol molecules are comparable in the two cases, i.e., $40 \mathrm{~kJ} \mathrm{~mol}^{-1}$ at the lower temperature and $38 \mathrm{~kJ} \mathrm{~mol}^{-1}$ at the higher temperature. The observed enhancement of desorption in the EMB experiments at higher temperatures may be attributed to the increased wettability by methanol on nopinone. This accelerates the monomer detachments from clusters, which may lead to an increase in desorption. Temperature increase eventually leads to cluster destruction, whereby the stronger interactions from $\sim 2$ hydrogen bonds conformations are vanishing and in turn make methanol monomers free to desorb from the nopinone surface. Thus, the surface acts as a boundary which interacts relatively weakly with methanol molecules but restrict the movements of trapped molecules.

\subsubsection{Liquid-Liquid Mixing}

As a substrate is kept below the melting point, the organic-organic system does not display any signs of mixing between the two organic species, even though one species could well wet the solid surface. The mixing of two liquid compounds is, however, interesting to investigate further. Due to technical and physical limitations of the current EMB setup it is not possible to experimentally investigate liquid-liquid interaction. A case of methanol cluster $(n=60)$
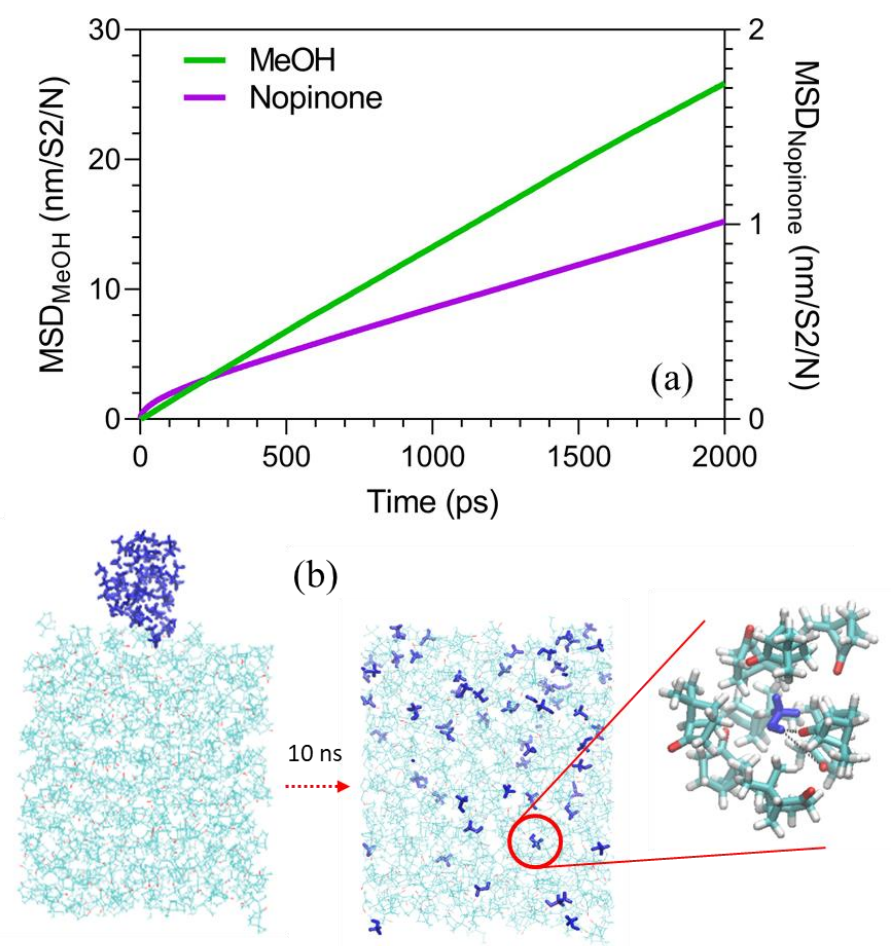

Figure 7 (a) Mean square displacement (MSD) evolution for nopinone and methanol during melting. (b) Snapshots of nopinone and methanol before and after melting in $10 \mathrm{~ns}$ simulation time. 
adsorbing on liquid nopinone surface is, however, simulated in MD. Liquid nopinone is obtained by applying simulated annealing all the way to $330 \mathrm{~K}$, and the nopinone bulk is then equilibrated at $270 \mathrm{~K}$. Figure $7 \mathrm{a}$ shows that the mean square displacements (MSD) of both methanol and nopinone are constantly growing over time at $270 \mathrm{~K}$. The increasing MSD of nopinone indicates that the liquid state of the nopinone, and the even steeper methanol MSD line is due to the fine mixing between the two compounds, with a methanol diffusion coefficient $D_{\text {methanol }}=(1.64 \pm 0.14) \cdot 10^{-5} \mathrm{~cm}^{2} \mathrm{~s}^{-1}$. Figure $7 \mathrm{~b}$ shows the initial and final snapshots, with an enhanced insert showing a methanol molecule and its neighboring nopinone molecules. Methanol clusters is dissolved in nopinone efficiently and methanol monomers are able to quickly find strong binding sites and make hydrogen bond with nopinone molecules (Figure 7b). The mixing ability between organics influences several key factors of the physiochemical properties of a mixture, such as the viscosity which in turn affects the gas particle partitioning of mixture components, and ice nucleation properties and heterogeneous chemistry of the system. ${ }^{39-41}$

\section{Conclusions}

The methanol-nopinone interactions are investigated by combining EMB experiments and MD simulations. Methanol monomers and clusters are sent to collide with graphite and nopinone coating/multilayer surfaces in the temperature range between $145 \mathrm{~K}$ and $230 \mathrm{~K}$. Methanol monomers are efficiently scattered from the graphite surface, and the scattering is significantly suppressed from nopinone surfaces, indicating a more significant energy transfer from the impinging molecules to the organic surfaces. When methanol clusters collide with graphite and nopinone surfaces, no significant differences in collision dynamics and desorption processes are observed between the surfaces, suggesting that the desorption is governed by methanolmethanol interactions rather than methanol-surface interactions. The MD results show that upon collisions the methanol clusters shatter, and the shattered fragments quickly diffuse and recombine to clusters. The desorption involves a series of processes, including detaching from clusters and desorbing from the nopinone surfaces as monomers. All trapped methanol molecules completely desorb within a short experimental time scale at the temperature of 180 $\mathrm{K}$ and above. At lower temperatures, the desorption rate decreases, and a long experimental time scale is used to resolve the desorption. Here three separate desorption components with different desorption rates are identified. The fast component is beyond the detection limit, and the desorption rates are not resolvable. The intermediate TD component exhibits a multi-step 
desorption character and has an activation energy of $E_{a}=0.18 \pm 0.03 \mathrm{eV}$, with a pre-exponential factor $A=10^{8.9 \pm 0.9}$. The slow desorption shows a weak temperature dependence, which indicates that it may relate to the diffusion process when methanol molecules exchange between the clusters. The MD results reveal that at lower temperatures methanol form compact cluster structures while at higher temperatures the methanol molecules form layered structures on the nopinone surface. Lastly, the MD simulation is used to study the liquid-liquid interaction, where the methanol clusters completely dissolve in the liquid nopinone, showing ideal organic-organic mixing.

\section{Acknowledgments}

This work is supported by the Swedish Research Council VR (2015-04212). XK acknowledges the supports from the National Natural Science Foundation of China (41975160) and the Swedish Foundation for International Cooperation in Research and Higher Education (CH2019-8361). This project has received funding from the European Research Council (ERC) under the European Union's Horizon 2020 research and innovation programme, Project SURFACE (Grant Agreement No. 717022).

\section{Conflicts of Interest}

The authors declare no conflict of interest.

\section{ORCID}

Xiangrui Kong: 0000-0002-7205-0723

Josip Lovric: 0000-0002-9618-9957

Sofia Johansson: 0000-0002-6139-3464

Nonne Prisle: 0000-0002-2041-6105

Jan B. C. Pettersson: 0000-0001-8420-6126

\section{References}

${ }^{1}$ D. M. Murphy et al., Journal of Geophysical Research: Atmospheres 111 (2006)

${ }^{2}$ G. McFiggans et al., Nature 565 (2019) 587.

${ }^{3}$ M. Kanakidou et al., Atmos. Chem. Phys. 5 (2005) 1053.

${ }^{4}$ M. Glasius, and A. H. Goldstein, Environ Sci Technol 50 (2016) 2754.

${ }^{5}$ A. Lee et al., Journal of Geophysical Research: Atmospheres 111 (2006)

${ }^{6}$ E. M. Neeman, J. R. Avilés-Moreno, and T. R. Huet, Physical Chemistry Chemical Physics 19 (2017) 13819.

${ }^{7}$ T. M. Cahill et al., Journal of Geophysical Research: Atmospheres 111 (2006)

${ }^{8}$ T. Suzuki, K. Ohtaguchi, and K. Koide, Computers \& Chemistry 16 (1992) 41.

${ }^{9}$ B. Nozière et al., Geophys. Res. Lett. 28 (2001) 1965.

${ }^{10}$ S. M. Johansson et al., J Phys Chem A 121 (2017) 6614. 
${ }^{11}$ S. M. Johansson et al., J Phys Chem A 124 (2020) 3652.

${ }^{12}$ X. Kong et al., Chemphyschem 20 (2019) 2171.

${ }^{13}$ L. F. Herrera, D. D. Do, and G. R. Birkett, Mol Simulat 36 (2010) 1173.

${ }^{14}$ X. Kong et al., J Phys Chem B 118 (2014) 13378.

${ }^{15}$ P. Papagiannakopoulos et al., Journal of Physical Chemistry C 117 (2013) 6678.

${ }^{16}$ X. Kong et al., J Phys Chem A 122 (2018) 4861.

${ }^{17}$ S. M. Johansson et al., Physical chemistry chemical physics : PCCP 21 (2019) 1141.

${ }^{18}$ E. S. Thomson et al., Atmos Chem Phys 13 (2013) 2223.

${ }^{19}$ E. S. Thomson et al., J Phys Chem Lett 2 (2011) 2174.

${ }^{20}$ P. Papagiannakopoulos et al., J Phys Chem B 118 (2014) 13333.

${ }^{21}$ X. R. Kong et al., Journal of Physical Chemistry C 116 (2012) 8964.

${ }^{22} \mathrm{X}$. Kong et al., in 12th International Conference on the Physics and Chemistry of Ice (PCI2010), edited by Y. Furukawa et al. (Hokkaido University Press, Sapporo, Japan, 2010), pp. 79.

${ }^{23}$ C. R. Arumainayagam, and R. J. Madix, Prog. Surf. Sci. 38 (1991) 1.

${ }^{24}$ B. A. C. Horta et al., J Chem Theory Comput 12 (2016) 3825.

${ }^{25}$ D. Van Der Spoel et al., J Comput Chem 26 (2005) 1701.

${ }^{26}$ A. K. Malde et al., J Chem Theory Comput 7 (2011) 4026.

${ }^{27}$ C. I. Bayly et al., The Journal of Physical Chemistry 97 (1993) 10269.

${ }^{28}$ L. Verlet, Phys Rev 159 (1967) 98.

${ }^{29}$ B. Hess et al., J Comput Chem 18 (1997) 1463.

${ }^{30}$ U. Essmann et al., The Journal of Chemical Physics 103 (1995) 8577.

${ }^{31}$ G. Bussi, D. Donadio, and M. Parrinello, The Journal of Chemical Physics 126 (2007) 014101.

${ }^{32}$ L. Palin et al., in Zeitschrift für Kristallographie International journal for structural, physical, and chemical aspects of crystalline materials2008), p. 602.

${ }^{33}$ C. R. Groom et al., Acta Crystallographica Section B 72 (2016) 171.

${ }^{34}$ N. Marković et al., Chem Phys 247 (1999) 413.

${ }^{35}$ M. B. Någård, N. Marković, and J. B. C. Pettersson, The Journal of Chemical Physics 109 (1998) 10350.

${ }^{36}$ M. B. Någård et al., The Journal of Chemical Physics 109 (1998) 10339.

${ }^{37}$ D. Schlesinger et al., The Journal of Physical Chemistry A (2020)

${ }^{38}$ E. Schröder, J Nanomater 2013, 871706 (2013) 1.

${ }^{39}$ J. P. Reid et al., Nat Commun 9 (2018) 956.

${ }^{40}$ T. Koop et al., Physical Chemistry Chemical Physics 13 (2011) 19238.

${ }^{41}$ T. Berkemeier et al., Atmos. Chem. Phys. 14 (2014) 12513. 